\title{
Mosquito biting and malaria situation in an urban setting in Zambia
}

\author{
Freddie Masaninga1, Daniel C. W. Nkhuwa ${ }^{2}$, Fastone M. Goma ${ }^{3}$, Cecilia Shinondo ${ }^{3}$, Emmanuel \\ Chanda $^{4}$, Mulakwa Kamuliwo ${ }^{4}$, Elizabeth Chizema Kawesha ${ }^{5}$, Seter Siziya ${ }^{6 *}$ and \\ Olusegun Babaniyi ${ }^{1}$
}

\author{
${ }^{1}$ World Health Organisation Country Office, Lusaka, Zambia. \\ ${ }^{2}$ School of Mines, University of Zambia, Lusaka, Zambia. \\ ${ }^{3}$ School of Medicine, University of Zambia, Lusaka, Zambia. \\ ${ }^{4}$ National Malaria Control Centre, Ministry of Health, Lusaka, Zambia. \\ ${ }^{5}$ Ministry of Health, Ndeke House, Lusaka, Zambia. \\ ${ }^{6}$ Public Health Unit, Clinical Sciences Department, The Copperbelt University, Ndola, Zambia.
}

Accepted 29 October, 2012

\begin{abstract}
Unprecedented increased mosquito bites were observed in urban and peri-urban areas of Lusaka District and there was a perceived increase in malaria which necessitated a study in 2009 to determine the mosquito biting and malaria situation within urban and peri-urban settings. We analysed water bodies as sources of mosquito larvae (vegetable gardens, sewerage maturation ponds and foot paths) and, weather factors for possible effects on mosquito densities, species distribution and reviewed laboratory confirmed malaria cases and interventions implemented in the previous five years from 2009. We collected high densities of Culex quinquefasciatus mosquito larvae (mean; 250 per scoop) from sewerage maturation ponds overgrown with water hyacinth - an aquatic vegetation and collected large numbers of adult Cx. quinquefasciatus indoor (mean: 14.2, range: 2.8 to 34 per room) and up to 2,000 Cx. quinquefasciatus out-door in one open structure. However, we did not find adult Anopheles in any of these collections but we found Anopheles larvae along a footpath in one peri-urban location. There was no evidence of increased malaria cases despite reported increased mosquito biting but a districtwide and nationwide decline in malaria trends. A combination of factors; human and environmental, created suitable micro-habitats that increased densities of the Culex mosquito but not malaria vector species within urban and peri-urban settings. An intensified surveillance, monitoring and evaluation measure is vital to understanding malaria situation and delivering effective malaria interventions in different epidemiological settings.
\end{abstract}

Key words: Mosquito, malaria, Culex quinquefasciatus, larvae, water hyacinth.

\section{INTRODUCTION}

Malaria is endemic in all the ten Provinces and has been a leading cause of mortality and morbidity in Zambia for several decades $(\mathrm{MOH}, 1988,1992,2000)$. Plasmodium falcipurum constitutes the most predominant malaria species $(98 \%)$ of all malaria infections in the Country with

\footnotetext{
${ }^{\star}$ Corresponding author. E-mail: ssiziya@gmail.com. Tel:
} 260955752646
$98 \%$ of total infections transmitted principally by Anopheles gambaie sensu latus and An. funestus $(\mathrm{MOH}$, 2010a). Malaria occurs throughout the year and transmission follows the main seasons; low transmission during the dry season from May to September and high transmission in wet hot season from November to April $(\mathrm{MOH}, 2005)$.

Culex mosquito species exist in Zambia. However, they do not constitute a major public health problem in Zambia as is the case with Anopheles vector species. Culex species 
are potential vectors of arthropod-borne viral (arboviral) infections in other ecological regions (Diaz-Badillo et al., 2011).

Zambia's national malaria control programme goal is to reduce the malaria incidence by $75 \%$ and all-cause child mortality by $20 \%$ of the 2010 baseline through a combination of interventions. The interventions being implemented are prompt diagnosis and effective treatment; Integrated Vector Management (using Long Lasting Insecticide Treated Mosquito Nets (LLINs), Indoor Residual Spraying (IRS) and environmental management in targeted areas; Malaria In pregnancy prevention; information, communication, education for Behaviour change communication and performance monitoring $(\mathrm{MOH}, 2006$, 2010b). Effective delivery of these interventions has contributed to a rapid positive impact on malaria. Inpatient routine health facility data shows a $30 \%$ reduction in malaria cases and deaths between 2006 and 2008 $(\mathrm{MOH}, 2008)$. These findings from national routine information system corroborate population surveys which show a decrease in parasite prevalence and severe anaemia (that is, haemoglobin less than $8 \mathrm{~g}$ per decilitre) in children below age five years, and severe anaemia reduced from $13.8 \%$ in 2006 to $4.3 \%$ in $2008(\mathrm{MOH}$, Malaria Indicator Survey, 2006, 2008).

Despite the national impact on the burden of malaria, communities in Lusaka reported that there was an increase in mosquito bites and perceived increased malaria cases in their residential areas from June to November, 2009. The World Health Organisation (WHO) in keeping with its core mandate of catalysing research supported the Ministry of Health (MOH) to determine the scope of the problem (WHO, 2008). The aim of this assessment was to investigate the underlying causes of the increased mosquito problem and to determine whether there was any increase in malaria cases in urban and peri-urban areas of Lusaka.

\section{METHODS}

\section{Searches for mosquito larval sites}

Searches for mosquito larvae were conducted in tertiary sewage ponds that is, the last ponds in the sewage treatment and settlement process; a dam, and in dug-out shallow wells within vegetable gardens in seven sites situated within urban and periurban residential areas of Garden Compound, Northmead, Mtendere, Kaunda Square, Matero, Ngwerere, and Chilenje South in Lusaka District (Figure 1). Of these residential areas, only one (Ngwerere) was more than $60 \mathrm{~km}$ away from the city centre of Lusaka. Coordinates were collected at each larval breeding site to use for mapping the areas for follow-ups. Surrounding areas in sewer ponds, disused vegetable gardening wells and a dam were searched for mosquito breeding using mosquito scoops (dippers). To scoop the mosquito larvae, the dippers were lowered within a depth of half a meter of the water bodies including; along the edges of a dam, sewer maturation ponds and garden wells. Larval densities were determined as the number of larvae per scoop.

Mosquito larvae were transferred to the Malaria Control Centre
Insectary and reared to the adult stage for identification.

\section{Sampling adult mosquitoes}

Adult mosquitoes were collected indoor from surfaces of 10 selected houses per day in the morning hours at 06.00 to $07.00 \mathrm{~h}$ in five residential areas (Northmead, Mtendere, Kaunda Square, Matero, and Ngwerere). The Spray sheet mosquito collection method was used for adult mosquito collections (WHO, 1992).

These collections were placed into containers labelled with information that included; location, date, time of collection, number of people per room. The mosquito samples were transferred on the same day to an entomology laboratory at the National Malaria Control Centre, Lusaka, for species identification (WHO, 1992; Gillies et al., 1968; Edwards, 1941). Uncompleted bathrooms and toilet constructions were sampled to assess if there could be any disparity between these and other indoor densities of the culicine mosquitoes. Mean densities (average number of mosquitoes resting indoors during the day) were used as a proxy measure for assessing the "biting threat" to the people living in residential areas from houses that were randomly selected.

\section{Review of meteorological information}

Meteorological data (maximum and minimum temperatures, rainfall and per cent relative humidity) for the period 2005 to 2009 was collected from the Meteorological Department database in Lusaka to assess weather patterns and trends, in order to ascertain any correlations with the problem of mosquito proliferation. The data is presented as average of three meteorological stations.

\section{Review of laboratory confirmed malaria cases}

We obtained laboratory malaria confirmed data for the period 2004 to $\mathbf{2 0 0 9}$ for children under the age five and above five years from a major referral centre (Matero) in Lusaka. Laboratory malaria blood microscopic examinations were conducted in accordance with the National Diagnosis and Treatment Policy ( $\mathrm{MOH}, 2010 \mathrm{~b})$. We also analysed trends and seasonal variations of the malaria parasite prevalance on the data maintained at the National Malaria Control Centre and from National surveys conducted in the period 2004 to 2009.

\section{Review of malaria interventions implemented in Lusaka district}

We reviewed Zambia Demographic and Health Survey (CSO et al., 2008), National Malaria Control Programme Reports and National Malaria Surveys carried out between 2004 and 2009 to gather information on malaria prevention and treatment interventions implemented in the district. Data included; ITN ownership and utilisation among different age categories (under five and 15 to 49 years pregnant women); IRS data from the National Malaria Programme reports and first line antimalarial medicine Artemether lumefantrine $(A L)$ from National Medical Stores Limited.

\section{Definitions}

IRS coverage is defined as proportion of households in target districts within urban and peri-urban sprayed within 12 months. Long Lasting Insecticide Treated Nets (LLINs) refers to factory pretreated nets for malaria control.

We visited four clinics; Matero main-referral centre, Chipata, 


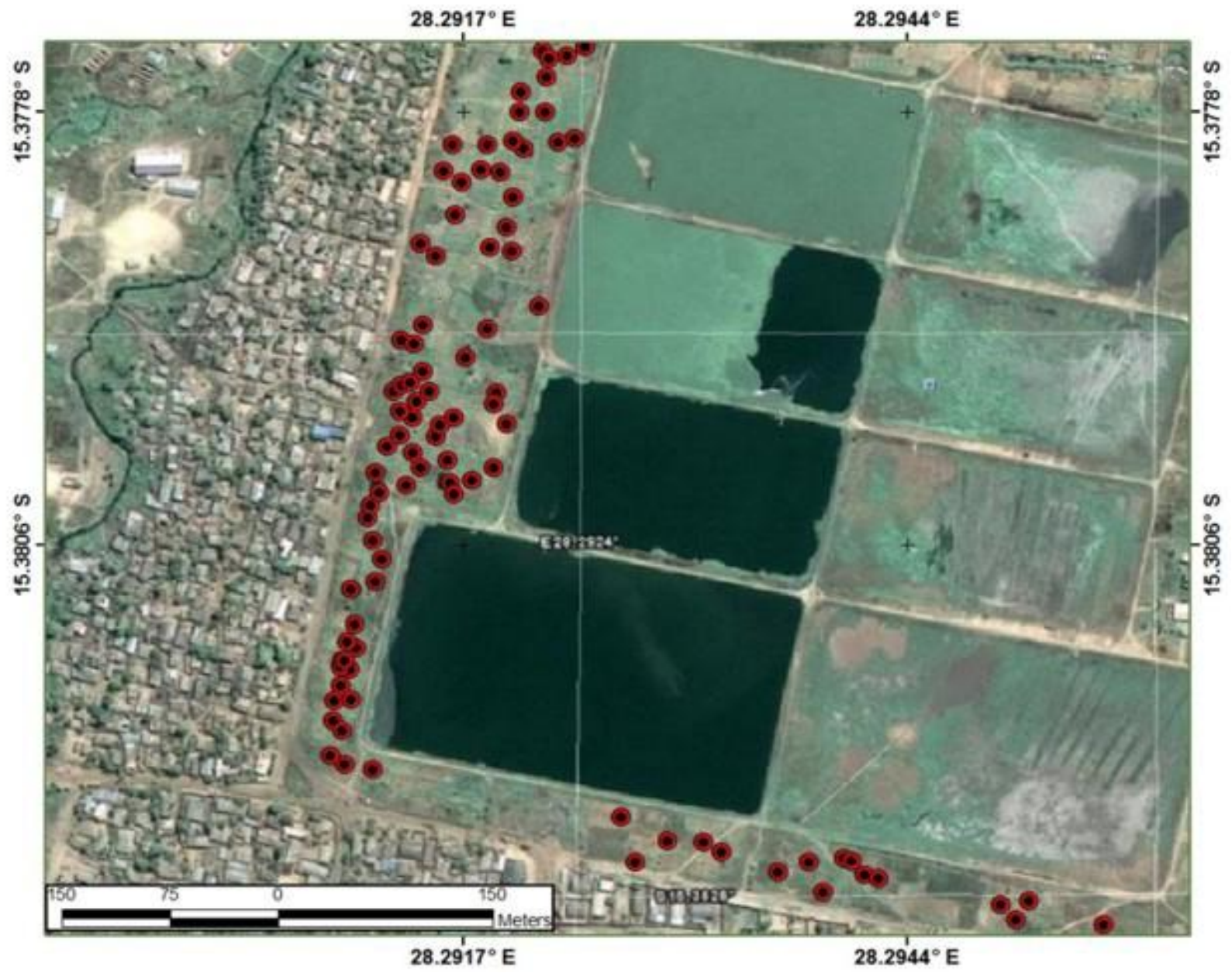

Figure 1. The scatter of shallow wells in vegetable gardens within maturation sewer ponds.

Source: Google Earth Maps.

Mtendere and Kaunda square to collect health worker perception on mosquito and malaria. At each of these health centres, five health workers, consisting of a medical doctor, a clinical officer and nurse were interviewed. The questionnaire guide requested the health worker(s) to comment on two main issues; (1) the mosquito and (2) malaria situation that is, to indicate whether there was an increase, decrease or no change regarding the mosquito and malaria situation in the past three years in their catchment areas and which antimalarial medicine was the recommended first line; its availability and efficacy. We verified availability of anti-malarials by checking the stock control cards in the pharmacy department. Each of the respondents interviewed had worked at their catchment area for at least three years.

\section{RESULTS}

\section{Search for mosquito larval sites}

Two hundred and fifty $C x$. quinquefasciatus species larvae (or more per scoop) were collected under an overgrown water hyacinth; aquatic vegetation and in maturation sewer ponds (Figure 2). These Culex mosquito larvae were collected along the edge of the water body in three township locations. Culicine larvae were also found in shallow wells within vegetable gardens located 5 to 10 $\mathrm{m}$ from sewer maturation ponds (Figure 2). However, larval counts were fewer in the garden wells (30 to 50 per scoop) than in maturation sewer ponds (250 Culex larvae per scoop). No Anopheles larvae of the malaria-carrying mosquitoes were collected in disused vegetable gardening wells, edges of a dam, stagnant water pools located between ridges, and tertiary sewer treatment ponds/sites sampled. However, scanty anopheles (ten larvae) and no culicine larvae were collected in one scoop from shallow (less than one meter deep) temporary water pool located in one peri-urban (Mtendere) along footpath, approximately five hundred meters away from a settlement site. These were reared at the laboratory; reared mosquitoes identified as Anopheles gambiae sensu latus.

\section{Sampling adult mosquitoes}

A total of 1,701 adult mosquitoes were caught -door during the entire three weeks study. The mean in-door 




Figure 2. Proliferation of the aquatic vegetation (hyacinth) in the sewage treatment ponds. The insert is a scoop showing the presence of larvae (appearing as dark spots) in the pond-water.

Source: Investigator, Nkhuwa DCN.

Perceptions on mosquito and malaria situation among health workers

resting density of these adult mosquitoes was 14.2 mosquitoes per room (Table 1). $80 \%$ of the female adult mosquitoes were fully fed (engorged). All the mosquitoes captured were identified into the $C x$. quinquefasciatus group; no adult Anopheles species were found in all the collections. The mean room occupancy was 3 people. A total of 321 people lived in the areas where mosquitoes were sampled. Culicine mosquitoes densities resting outdoor were too numerous to count. Approximately 2,000 adult $C x$. quinquefasciatus were collected in a small (two metres square) bathroom adjoining a house which constituted the highest count within the five residential areas sampled. A similar high density of at least 170 culicine mosquitoes and more were collected from a small disused broken cement drain leading to treatment sewerage ponds, measuring two square metres, situated approximately $5 \mathrm{~km}$ from one residential area (Kaunda square) (Figure 3). Favoured adult mosquito resting sites included hyacinth vegetation in the sewage maturation ponds inside pit latrines and dug-out garden wells.

\section{Review of meteorological information}

Temperature, rainfall and relative humidity trends in Lusaka are shown in Figure 4. The data are averages of three Meteorological Stations (Lusaka International Airport, City Airport and Mount Makalu) collected between July 2005 to September 2009.

\section{Review of laboratory confirmed malaria cases}

Annual confirmed malaria cases showed a decreasing trend in Lusaka district for both children under the age of five and those above five (that is, all malaria cases) since 2004 with slide positivity at $16 \%$ in 2005 and $1.5 \%$ in 2009 (Figure 5). MOH, Malaria Indicator Survey (2006, 2008) showed a similar trend in parasite prevalence data for children under the age of five years (22\%) in 2006 compared with $10 \%$ in 2008.

\section{Malaria interventions in Lusaka district}

The number of formal housing structures sprayed in Lusaka 
Table 1. Adult culicine mosquito resting densities and larvae from breeding sites in five residential areas, November, 2009, Lusaka, Zambia.

\begin{tabular}{lcccc}
\hline Locality (urban or peri-urban) & Mean mosquitoes collected per room & Rooms sampled & Water source & Larvae per dip \\
\hline Garden (Peri-U) & 34.00 & 20 & Sewer ponds & +250 \\
North Mead (Urban) & 2.80 & 20 & Sewer ponds & +100 \\
Mtendere (Peri-U) & 6.87 & 15 & $*$ & 0 \\
Kaunda Sq. (Urban) & 29.68 & 28 & Sewer ponds & +250 \\
Chilenje (Peri-U) & 4.00 & 14 & $*$ & 0 \\
Average & 14.20 & & & \\
\hline
\end{tabular}

*No sewer ponds were available for larval searches in Mtendere and Chilenje sites. However, Anopheline larvae mosquitoes were collected in periurban areas in Mtendere areas from a shallow water pool. (Abbreviation; peri-U = peri-urban).

Source: Data collected by PI - FM and other investigators CS.

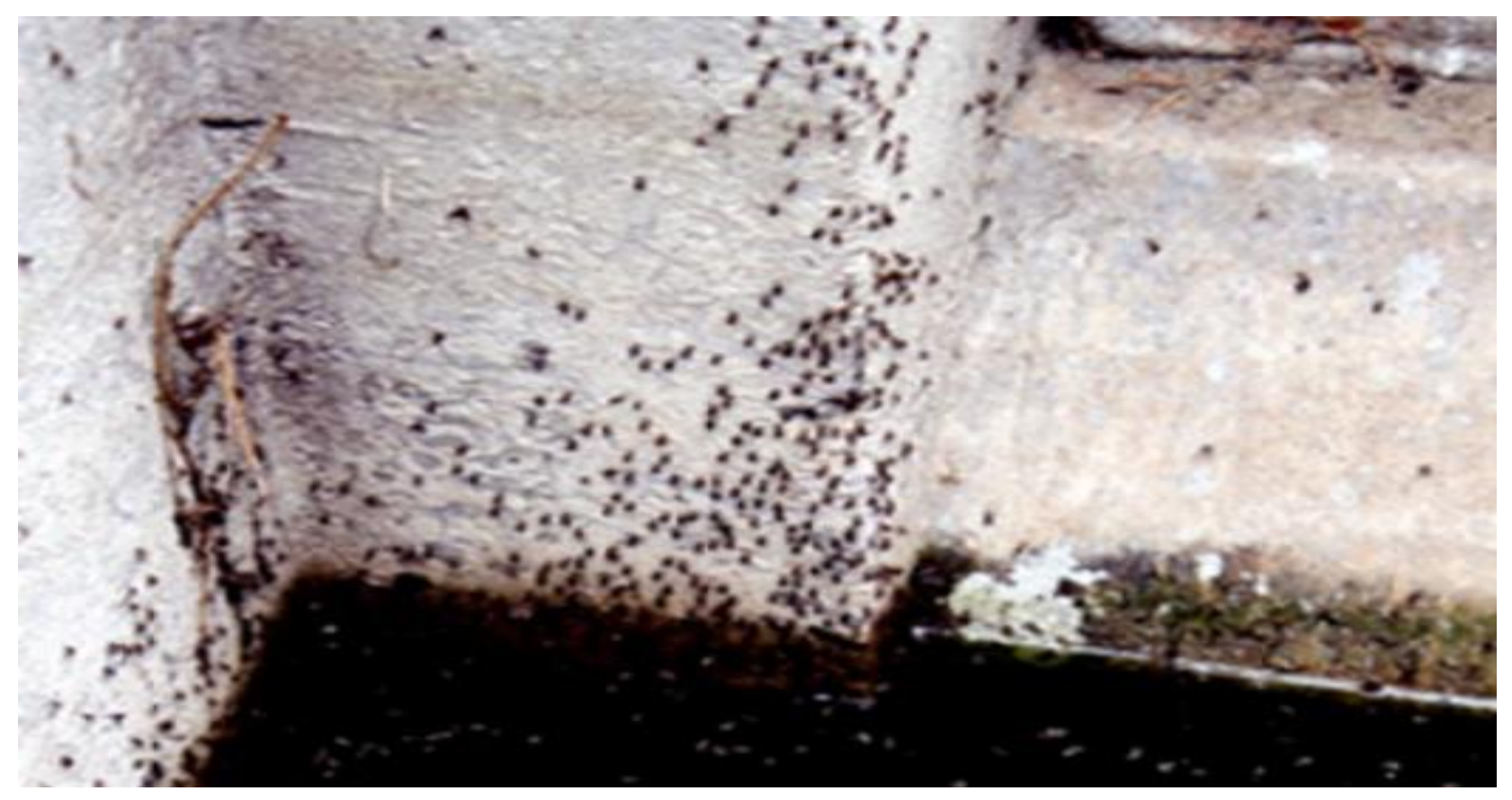

Figure 3. Mosquitoes' hideouts disused manholes, located five meters from sewer ponds at Kaunda Square. Adult mosquitoes appear as black spots.

Source: Principal Investigator (PI), Masaninga F.

Lusaka District increased from 7,000 in 2003 to 322,000 structures in 2008 while the informal structures increased from 8,800 to 85,000 . The IRS coverage in Lusaka district was $11 \%$ in 2006 and $30 \%$ in 2008. Likewise, the national IRS coverage was $6.5 \%$ in 2005 and $42 \%$ in 2008. Organochlorine (DDT) and pyrethroids insecticides (alphacypermethrin, lambdacyhalothrin and K-orthrine) have been used for indoor spraying since the reintroduction of IRS in 2003. DDT has been targeted to rough, non-plastered wall surfaces while pyrethroids targeted smooth cement-plastered surfaces. National ITNs data for the period 2006 to 2008 showed increased national household ownership from $44 \%(\mathrm{~N}=2957)$ to $62 \%(\mathrm{~N}=4,405)$. Likewise Lusaka district ITN use increased among children of age below five years from
$15 \%(N=321)$ to $42 \%(N=363)$ and in pregnant women from 11 to $46 \%$. ITN utilisation by wealth index showed a similar trend; an increase in the lowest quintile from $22 \%$ $(\mathrm{N}=581)$ to $39 \%(\mathrm{~N}=1,082)$ for children and from $21 \%$ $(\mathrm{N}=88)$ to $40 \%(\mathrm{~N}=103)$ for pregnant women. The increasing trend in ITN use was true for the highest wealth index as well; increasing from $30 \%(\mathrm{~N}=408)$ to $40 \%(\mathrm{~N}=611)$ in children and from $25 \%$ in $2006(\mathrm{~N}=75)$ to $49 \%$ in $2008(\mathrm{~N}=416)$ in pregnant women. National distribution of $A$ lumefantrine by the Medical Store Limited averaged 2.1 million treatment courses in the period 2004 to 2009 (Figure 6). The average annual distribution of the AL treatment courses for all the age groups for Lusaka district was 291,000 (range: 292,882 to 318,574$)$. 


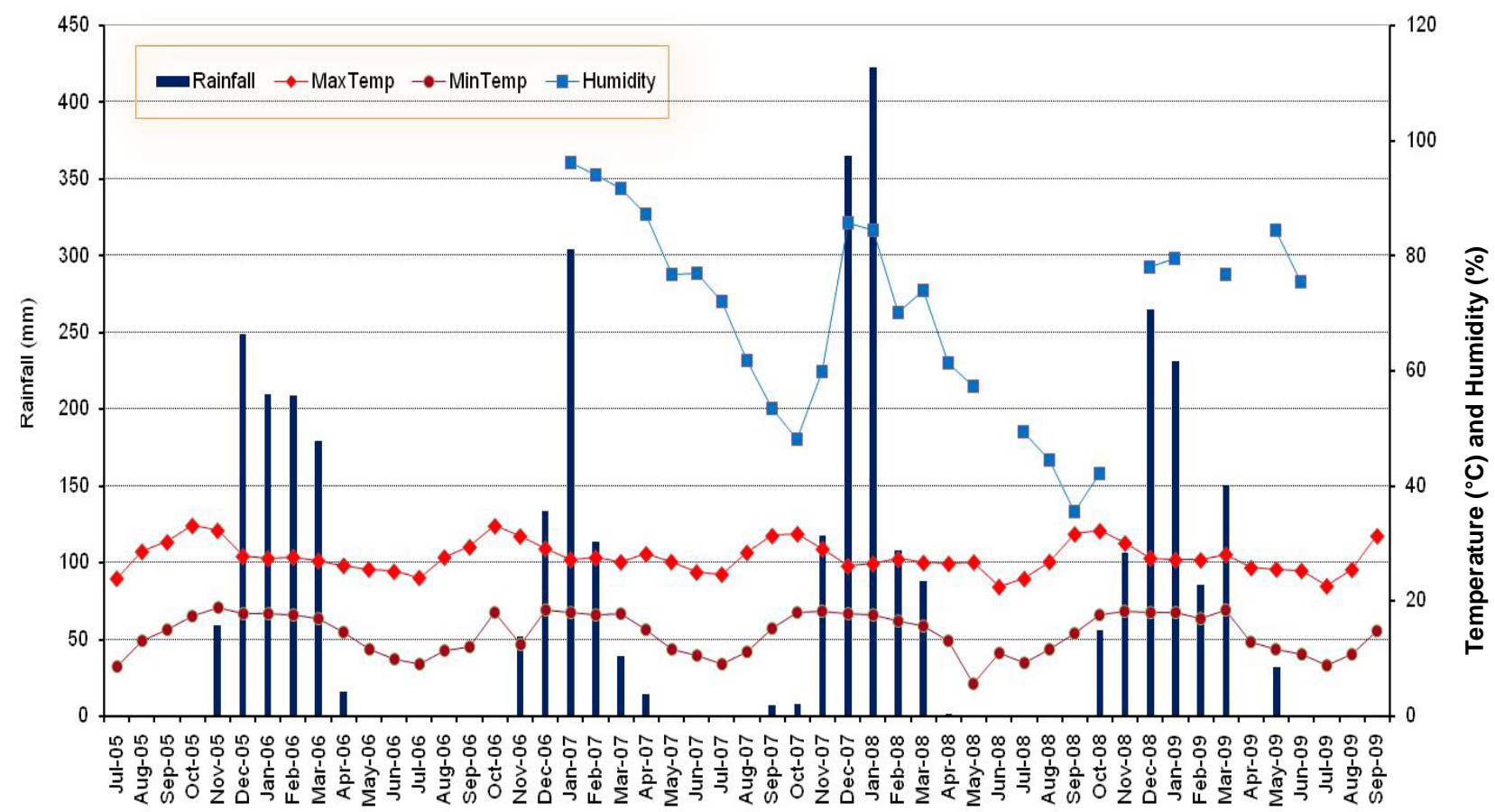

Figure 4. Recent trends in rainfall, maximum and minimum temperatures and relative humidity for Lusaka as averages of the three Meteorological stations, namely; Lusaka International Airport, City Airport and Mount Makulu between 2005 to 2009. Source: National Meteorological Department, Lusaka, Zambia.

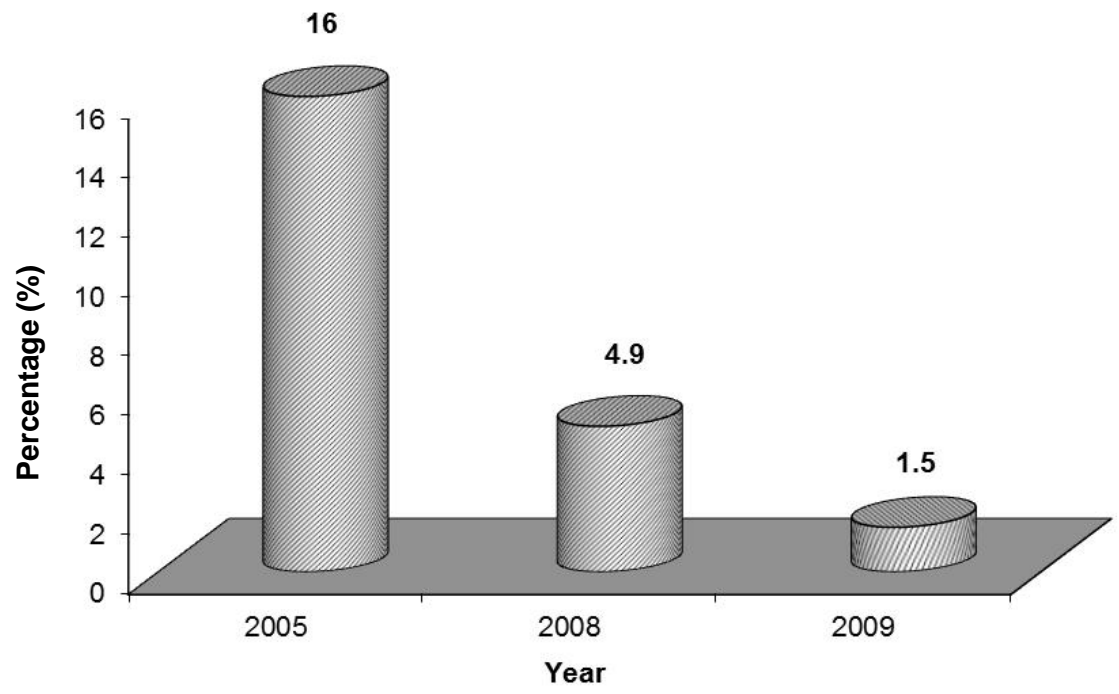

Figure 5. Percent slide positive tested by microscopy for Plasmodium falcipurum malaria cases by year, Mtendere Clinic, Zambia (Number. tested $=4934$ in 2005; 10,043 in 2008; 8,314 in 2009).

Source: Mtendere Clinic, Ministry of Health, Lusaka Zambia.

\section{Perceptions on mosquito and malaria situation among health worker}

All the 20 health workers interviewed indicated that the number of mosquitoes biting them during the late afternoon into the evenings was a record high, when compared to the past three years and high biting occurred even in the dry-cool season (June). However, interviews indicated an apparent decrease in malaria cases within their catchment health facilities (clinics) during these past 


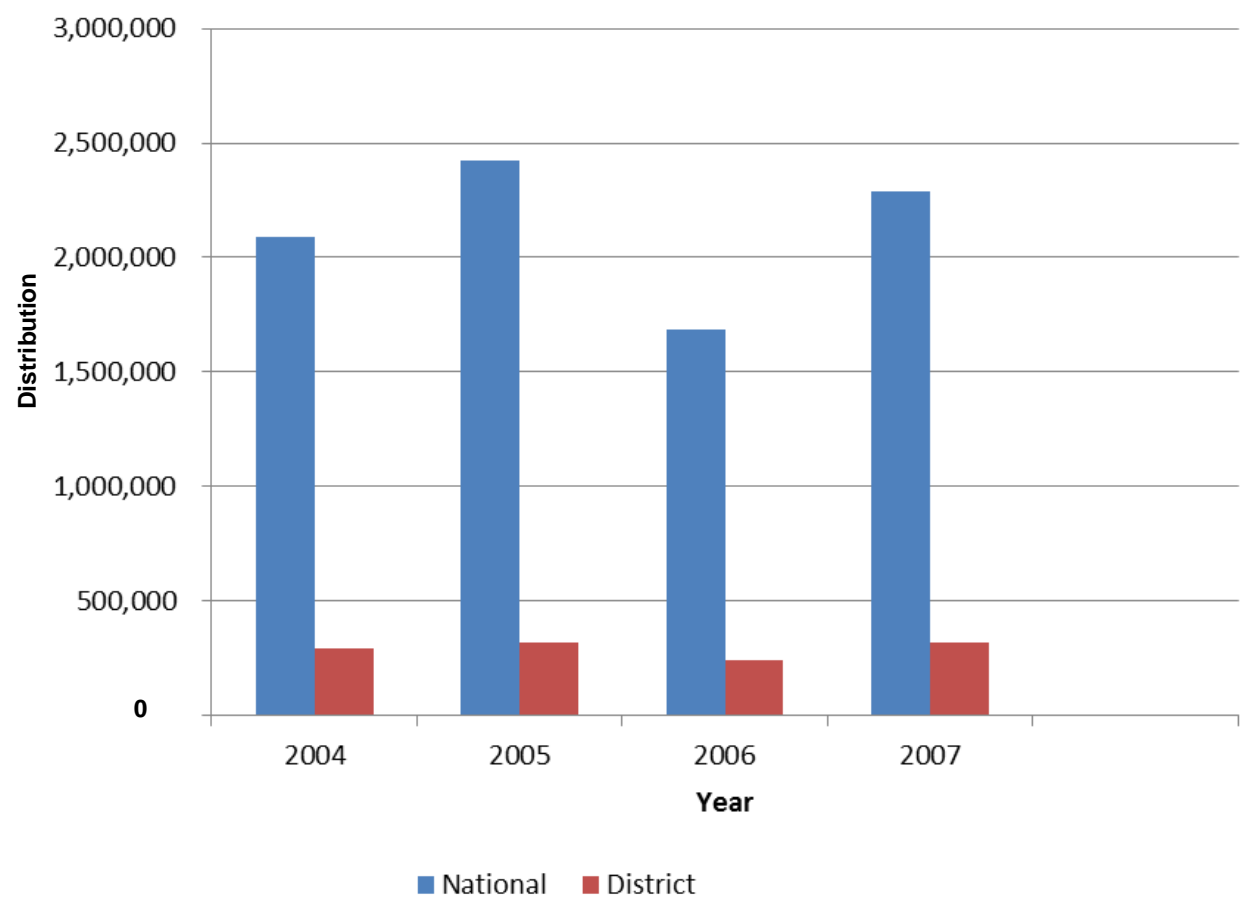

Figure 6. National compared to District (Lusaka) distribution of the first line (Artemether lumefantrine) 2004 to 2007.

Source: National Malaria Control Centre and Medical Stores Limited, Lusaka, Zambia.

three years. They reported Artemether lumefantrine to be the recommended first line anti-malarial medicine for the treatment of uncomplicated malaria, and that it was generally available, providing adequate therapeutic cure, with no recorded treatment failure.

\section{DISCUSSION}

There was no evidence of increased malaria cases as perceived by the communities in the Lusaka environment, in the period under review but on the contrary, a declining trend of $P$. falcipurum malaria slide positivity occurred in Lusaka between 2004 and 2009. This finding corroborates with the nation's Ministry of Health $(\mathrm{MOH})$ Management Information System. This routine system has recorded declines in-patient malaria cases and deaths of 46 and 47\%, respectively between 2006 and 2008. Similarly, WHO reported national declines of 55 and $60 \%$ in 2008, when the 2008 malaria data was compared to the 2001 to 2002 reference point (WHO, 2009; WHO Zambia Country Office, 2009); this decline continued to be reported (WHO, 2010). Nationally, representative malaria surveys report a general decline in $P$. falcipurum parasite prevalance in children of age 0 to 59 in Zambia (MOH, Malaria Indicator Survey, 2006, 2008).

This assessment on malaria-mosquito situation in Lusaka showed scarce anopheline malaria vector breeding in the areas sampled. The observed paucity of malaria-transmitting-anopheles vectors in the city corroborates the local declines in the malaria situation, particularly for the month of November when sampling was conducted. The apparent absence of adult Anopheles malaria vectors show changes in the epidemiology of vectors that may be related to increased city expansion that has occurred in the last four years. This may have contributed significantly to the destruction of mosquito breeding sites. Furthermore, interventions such as ITNs, IRS and Artemisinin combination therapy (ACTs) for treatment of uncomplicated malaria have significantly been scaled-up above the national and regional targets (Abuja Declaration and the Plan of Action, 2000; MOH, 2010a; WHO, 2009; Barnes et al., 2009). Continued vector and disease surveillance are vital to guide effective delivery of preventive, diagnostics and treatment interventions $(\mathrm{MOH}, 2002,2010 \mathrm{~b}$; WHO, 2010; 2004).

High densities of culicine mosquitoes were observed outdoors in sewerage treatment ponds overgrown with water hyacinth, disused holding holes, water drains and in pit latrines. Breeding sites were situated close to homes; within a distance of 10 to $20 \mathrm{~m}$. Socio-economic activities, including vegetable gardening located 5 to 10 $\mathrm{m}$ away from the sewage ponds contributed to the proliferation of mosquito populations during the dry season, when these gardening or vegetable growing activities increased. Health workers at different health facilities confirmed the unprecedented increased numbers of 
mosquitoes biting people that were experienced starting in the afternoon around 16.00 to $17.00 \mathrm{~h}$ which we identified as $C x$. quinquefasciatus. $C x$. quinquefasciatus transmits filariasis and viral infections in African and other regions (WHO, 2001; Service, 1986).

WHO disease distribution maps show the occurrence of filariasis of up to $11 \%$ prevalence on the Copperbelt Province and $6 \%$ in the North-Western Province of Zambia (WHO Zambia Country Office, 2009). Therefore, our findings highlight the need to develop comprehensive vector control strategies for malaria and other mosquitoborne diseases and to enforce relevant Public Health Act to promote a healthier environment (Mosquito extermination Act, CAP 537, 1964; Laws of Zambia, 1964).

Eighty percent of the female culicine mosquitoes collected indoors were blood-fed. Even though no tests were done to determine the source, the blood was most likely human, given the paucity of domestic animals in the area. That the public complaints about mosquitoes occurred in winter and daytime biting was a clear indication that the biting was unlikely to be related to malaria.

In the 1960s and early 1970s, there was limited breeding of mosquitoes in the city of Lusaka, with no or occasional bites around households; malaria was also a notifiable disease $(\mathrm{MOH}, 2002)$. Intensive surveillance ensured early response to even a single reported case in this and other cities. During this period, there were fewer unplanned settlement residential areas and relevant statutory instruments were enforced through the local government which obliged each household to maintain hygiene standards and to prevent and control mosquito breeding. However, mosquito breeding and malaria cases increased during the late 1970s to 1990 s due to worsening socio-economic factors concomitant with rapid urbanisation; this urbanisation has continued to expand in the major cities in Zambia $(\mathrm{MOH}, 1992,2000$; UN Habitat, 2012).

Weather data in this study suggest an increase in total seasonal rainfall for 2007 to 2008 and 2008 to 2009 seasons compared to the preceding years. However, there was no apparent change in temperature patterns. These and meteorological data on relative humidity were insufficient to make inference on mosquito occurrence. The changing malaria epidemiology and sporadic increases in culicine mosquito density, low numbers of both confirmed malaria cases and malaria vectors necessitate effective interventions in Lusaka including; Integrated Vector Management, Systematic monitoring of vector and malaria case investigation, strengthened community participation and adherence to legal provisions to prevent mosquito breeding and malaria proliferation in the city.

\section{Conclusion}

Decreasing malaria prevalence in Lusaka environment following effective anti-vector measures (IRS and ITNs), diagnosis and malaria treatment necessitates an intensification of surveillance, monitoring and evaluation to sustain the impact on the disease but also to strengthen understanding of the malaria-mosquito epidemiology to curb future proliferation of nuisance mosquitoes.

\section{REFERENCES}

Abuja Declaration and the Plan of Action (2000). An extract from the African Summit on Roll Back Malaria, Abuja, $25^{\text {th }}$ April (WHO/CD/RBM/2000.17). [On line] http://www.rollbackmalaria.org/docs/abuja_declaration_final.htm.

Barnes KI, Chanda P, Barnabas GA (2009). Impact of large scale deployment of artemether lumefantrine on malaria disease burden in Africa: case studies of South Africa, Zambia and Ethiopia. Malar. J. 8(Suppl 1):S8.

Diaz-Badillo A, Bolling BG, Perez-Ramirez G, Moore CG, MartinezMunoz JP, Padilla_Viveros AA, Camacho-Nuez M, Diaz-Perez A, Beaty BJ, Munozi M (2011). The distribution of potential West Nile virus vectors, Culex pipiens pipiens and Culex pipiens quinquefasciatus (Diptera: Culicidae), in Mexico City. Parasit Vectors $9 ; 4: 70$.

CSO, MOH, TDRC, UNZA, Macro International (2008). Zambia Demographic and Health Survey 2007. Preliminary report. Calverton, Maryland, USA: CSO and Macro International Inc. [On line] http://www.africanchildinfo.net/documents/Zambia\%20DHS\%202007 \%20PRELIMINARY\%20REPORT.pdf

Edwards FW (1941). Mosquitoes of the Ethiopian Region, III - Culicine Adults and Pupae. British Museum (Natural Museum), London.

Gillies MT, De Meillon B (1968). The Anophelinae of Africa south of the Sahara: Ethiopian Zoographical Region, $2^{\text {nd }}$ ed. Johannesburg: South African Institute for Medical Research.

Malaria Indicator Survey (MIS) 2006. Zambia Ministry of Health, Zambia Central Statistical Office, PATH, MACEPA, CDC, WHO. Lusaka: Ministry of Health. [Online]. Available from: http//nmcc.org.zm.whites.net/files/2006_Zambia_Malaria_Indicator_S urvey.pdf. [Accessed on 24 November, 2011].

Malaria Indicator Survey (MIS) 2008. Zambia Ministry of Health, Zambia Central Statistical Office, PATH, MACEPA, CDC, WHO. Lusaka: Ministry of Health. Online Available from: http://www.nmcc.org.zm/files/ZambiaMIS2008Final.pdf. [Accessed on 24 November, 2011].

Ministry of Health, MOH (1988). Bulletin of Health Statistics. 19871988. Major Health Trends in 1987-1988. Health Information Unit. Ministry of Health, Lusaka, Zambia.

Ministry of Health, MOH (1992). Bulletin of Health Statistics. 1989-1992. Major Health Trends in 1982-1992. Health Information Unit. Ministry of Health, Lusaka, Zambia.

Ministry of Health, MOH (2000). National Malaria Control Programme. Malaria Situation analysis. Lusaka. Zambia: Ministry of Health.

Ministry of Health, MOH (2002). Technical Guidelines for Integrated Disease Surveillance and Response (IDSR) in Zambia. Adapted from Technical Guidelines for IDSR in the African Region developed by WHO AFRO and CDC. Ministry of Health, Lusaka, Zambia

Ministry of Health, MOH (2005). National Malaria Control Programme; 2006-2010. National Malaria Strategic Plan. Lusaka. Zambia: Ministry of Health.

Ministry of Health, MOH (2006). 3-Year Implementation Plan, 20062008. A Road Map for Impact on malaria in Zambia. Rapid Scale-up of malaria control interventions for impact. Lusaka, Zambia: Ministry of Health. [On line] http://www.nmcc.org.zm/files/NMCC3YearlmplementationPlanZMOH. doc.

Ministry of Health, MOH (2008). Annual Health Statistical Bulletin. Lusaka. Zambia. Ministry of Health.

Ministry of Health, MOH (2010a). Malaria Performance Review, 2010. Ministry of Health Zambia, National Malaria Control Centre. Lusaka. Zambia.

Ministry of Health, MOH (2010b). Guidelines for the diagnosis and treatment of malaria in Zambia. 3rd edition. Lusaka, Zambia. 
Ministry of Health Mosquito extermination Act, CAP 537 (1964). Laws of Zambia, 1964.

Service MW (1986). Culicine Mosquitoes. In: Lecture Notes on Medical Entomology. London: Blackwell Scientific Publications: 59-61.

Habitat UN (2012). Nations Human Settlement Programme. UN Habitat for better future in Zambia. Zambia Urban Housing Sector Profile. June 2012. [On line] http://www.scribd.com/doc/101018896/ZambiaUrban-Housing-Sector-Profile

World Health Organisation, WHO (1992). Entomological Field Techniques for malaria control, Part 1. Learner's Guide. World Health Organisation, Geneva.

World Health Organisation, WHO (2001). Global Programme to eliminate Lymphatic Filariasis. Annual Report on Lymphatic Filariasis. Geneva: World Health Organisation. WHO/CDC/CPE/CEE/2002.28.

World Health Organisation, WHO (2004). Global Strategic Framework for Integrated Vector Management. 2004. World health Organisation, Geneva.

http://whqlibdoc.who.int/hq/2004/WHO_CDS_CPE_PVC_2004_10.pd f
World Health Organisation, WHO (2008). Engaging for Health. Eleventh General Programme of Work, 2006-2015: A Global Agenda. World Health Organisation, Geneva.

World Health Organisation, WHO (2009). World Malaria Report 2008. World Health Organization, Geneva. (WHO/HTM/GMP/2008.1). [On line]

http://www.who.int/malaria/world_malaria_report_2009/en/index.html.

World Health Organisation, WHO Zambia Country Office (2009). Strengthening the health system in Zambia. Annual report of the WHO Country Office in Zambia. Lusaka, Zambia: WHO Country Office in Zambia.

World Health Organisation (2010). World Malaria Report 2010. WHO, Geneva.

[Online]. http://www.who.int/malaria/world_malaria_report_2010/worldmalariar eport2010.pdf. 